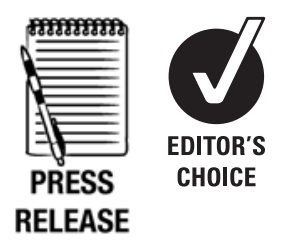

Division of Primary Care, Nottingham, UK

\section{Correspondence to}

Dr Julia Hippisley-Cox, Division of Primary Care, 13th floor,

Tower Building, University Park, Nottingham NG2 7RD, UK; julia.hippisley-cox@nltworld. com

Data Sharing statement The patient level data from the QResearch and THIN databases are specifically licensed according to the governance framework for each database. See http://www.qresearch.org for further details.

Algorithms and software The algorithms presented in this paper will be released as OpenSource Software under the GNU lesser GPL v3. The web calculator can be found at http://www.qintervention.org.

Accepted 30 March 2010

\title{
Individualising the risks of statins in men and women in England and Wales: population-based cohort study

\author{
Julia Hippisley-Cox, Carol Coupland
}

\begin{abstract}
Objective To derive and validate risk algorithms so that the risks of four clinical outcomes associated with statin use can be estimated for individual patients.

Design Prospective open cohort study using routinely collected data from 368 QResearch general practices in England and Wales to develop the scores. The scores were validated using two separate sets of practices-188 separate QResearch practices and 364 practices contributing to the THIN database.
\end{abstract}

Subjects In the QResearch derivation cohort 225922 new users of statins and 1778770 non-users of statins were studied. In the QResearch validation cohort 118372 statin users and 877812 non-users of statins were studied. In the THIN validation cohort, we studied 282056 statin users and 1923840 non-users of statins were studied.

Methods Cox proportional hazards models in the derivation cohort to derive risk equations. Measures of calibration and discrimination in both validation cohorts. Outcomes 5-Year risk of moderate/serious myopathic events; moderate/serious liver dysfunction; acute renal failure and cataract.

Results The performance of three of the risk prediction algorithms in the THIN cohort was very good. For example, in women, the algorithm for moderate/serious myopathy explained $42.15 \%$ of the variation. The corresponding D statistics was 1.75 . The acute renal failure algorithm explained $59.62 \%$ of the variation (D statistic $=2.49$ ). The cataract algorithm explained $59.14 \%$ of the variation (D statistic $=2.46$ ). The algorithms to predict moderate/severe liver dysfunction only explained $15.55 \%$ of the variation (D statistics $=0.89$ ). The performance of each algorithm was similar for both sexes when tested on the QResearch validation cohort.

Conclusions The algorithms to predict acute renal failure, moderate/serious myopathy and cataract could be used to identify patients at increased risk of these adverse effects enabling patients to be monitored more closely. Further research is needed to develop a better algorithm to predict liver dysfunction.

\section{BACKGROUND}

Cardiovascular disease is the leading cause of premature death and a major cause of disability in the UK. ${ }^{1}$ The better identification and management of high-risk patients is a major priority. ${ }^{2}$ Validated cardiovascular risk prediction algorithms, such as ORISK2, ${ }^{3}$ are used to identify high-risk patients most likely to benefit from interventions including smoking cessation, weight reduction, blood controls and lipid modification using statins. ${ }^{5}$

The implementation of the Department of Health Vascular Screening Programme ${ }^{2}$ could result in an extra 1.8 million patients starting long-term statin treatment. ${ }^{5 a}$ While the benefits of statins are relatively well established for patients at high risk of cardiovascular disease, a number of studies have found that patients starting statins are at increased risk of adverse effects, including liver dysfunction, ${ }^{5 \mathrm{a}} 7$ myopathy, ${ }^{5-9}$ acute renal failure ${ }^{67}$ and cataracts. ${ }^{5 a}$

In order to consent to treatment, patients need information on their baseline cardiovascular risk and how that would be reduced with interventions. They also need information on the risks and benefits of interventions as indicated in both the British National Formulary and NICE guidelines on lipid modification. ${ }^{11}$ Utilities are therefore needed to individualise risk estimates for patients so that this information can be discussed during the consultation. Clinicians also need to identify those at greatest risk of adverse events so that they can be more closely monitored.

In this paper, we develop and validate four new risk prediction algorithms to quantify an individual's absolute risk of an adverse clinical outcome which is likely to change with statin use. The outcomes included are cataract, moderate/serious myopathy, moderate/serious liver dysfunction and acute renal failure, which we found to be associated with statin use in a companion paper. ${ }^{5 a}$ As with previous algorithms, ${ }^{3} 1213$ we have used readily available data held within primary care electronic health records as this is both generalisable to the general population and can be integrated back into the same clinical setting for use at the point of care.

\section{METHODS}

\section{Study design and data source}

We carried out a prospective cohort study in a large population of primary care patients from an open cohort study using the OResearch database (version 24). We included all practices in England and Wales which had been using their EMIS computer system for at least a year. We randomly allocated twothirds of practices to the derivation dataset and the remaining third to a validation dataset. We identified an open cohort of patients aged 30-84 years drawn from patients registered with practices between 01 Jan 2002 and 30 June 2008. We excluded patients who did not have a postcode-related Townsend score (about 4\% of the population) and those who had been prescribed statins before the study start date. Entry to the cohort was the latest of study start date, 12 months after the patient registered with the practice or date of first statin prescription. Patients were censored at the earliest date of the diagnosis of interest, death, deregistration with the practice, last upload of computerised data or the study end date (31 December 2008). 
We used an identical sample of patients drawn from practices contributing to the validated THIN database, although the study end date for this sample was 30 June 2008, as an independent and external validation dataset.

\section{Clinical outcomes}

We examined the following outcomes as these outcomes were found to be associated with statin use in our previous paper: ${ }^{5 a}$

- Acute renal failure (from relevant Read codes recorded during follow-up).

- Moderate or serious liver dysfunction defined as alanine transaminase >120 IU/1 (ie, more than three times upper limit of normal) among patients without diagnosed chronic liver disease at baseline as this is the severity at which guidelines recommend that treatment is discontinued. ${ }^{11}$

- Moderate or serious myopathic events, ${ }^{8}$ 14-16 which for our study were defined as a diagnosis of myopathy or rhabdomyolysis or raised creatine kinase of four or more times the upper limit of normal (>560 in women and $>696$ in men) as this represents an event where treatment is likely to be discontinued.

- Cataract (from relevant Read codes recorded during followup)

Using the trials reported in a meta-analysis ${ }^{5}$ of statins in patients with risk factors for cardiovascular disease, we combined the results for major coronary events and cerebrovascular events using a random effects model to calculate a summary relative risk (RR) for cardiovascular disease only including trials which reported both outcomes and assuming that individual patients did not have both outcomes. We combined this RR with the QRisk2 cardiovascular disease score ${ }^{3}$ to estimate the risk of cardiovascular disease for an individual taking statins

\section{Risk factors and statin exposure}

As described in detail in the companion paper, ${ }^{5 a}$ we identified new users of statins during the study period, and excluded current and past users at baseline, with the remaining patients classified as non-users of statins. We compiled a long list of potential predictor variables which included statin use and established risk factors for each disease from the literature or existing risk prediction scores using similar definitions where possible.

\section{Derivation and validation of the models}

We developed and validated the risk prediction algorithms using established methods. ${ }^{3} 91317-19$ We used multiple imputation to replace missing values for body mass index, and smoking status and used these values in our main analyses. ${ }^{20-23}$ We carried out five imputations. We used Cox's proportional hazards models to estimate the coefficients for each risk factor for men and women separately, using Rubin's rules to combine the results across the imputed datasets. We used fractional polynomials to model nonlinear risk relationships with continuous variables. ${ }^{24}$ We included statins as a binary variable since our previous paper indicated no substantial difference between risk of most outcomes by type or dose of statin. ${ }^{5 a}$ We used backward selection procedures to eliminate variables using a $p$ value of 0.01 to obtain parsimonious models.

We examined interactions between predictor variables and age, and we included significant variables and significant interaction terms in the final models. We took the regression coefficients for each variable from the final models and used these as weights which we combined with the baseline survivor function for each outcome evaluated at 5 years to derive risk equations at 5 years' follow-up.

We used multiple imputation in both validation cohorts to replace missing values for body mass index and smoking. We then applied the algorithms obtained from the derivation cohort to both validation cohorts and calculated measures of discrimination (D statistic ${ }^{25}$ and area under the receiver operating characteristic curve (ROC statistic)) and calibration (comparing observed with predicted risks by tenth of predicted risk). We applied each risk score to the OResearch validation cohort to define the threshold for the top $10 \%$ of patients at risk of each adverse event.

We used the THIN validation sample for our main validation as this is from practices using a different clinical computer system from that of OResearch practices. We used all the available data on each database to maximise the power and also generalisability of the results. We used STATA (version 11) for all analyses.

\section{Numbers needed to treat/harm}

As described in the companion paper in the $B M J,{ }^{5 \mathrm{a}}$ we also calculated the numbers needed to treat (NNT) or harm (NNH) for each outcome over 5 years for patients at high risk of cardiovascular disease based on a ORISK 2 score of $\geq 20 \%$, since this high-risk group is eligible for statin treatment. We also calculated the number of additional cases per 10000 patients treated over 5 years to enable comparisons and supplement data published elsewhere.

\section{RESULTS}

\section{Overall study population}

Overall, 557 OResearch practices in England and Wales met our inclusion criteria, of which 369 were randomly assigned to the derivation dataset with the remainder assigned to a validation cohort. We identified 2121786 patients aged 30-84 years in the QResearch derivation cohort. We excluded 9513 (0.45\%) past users of statins and $107581(5.07 \%)$ current users, leaving 1778770 patients $(83.83 \%)$ who had not been prescribed statins and $225922(10.65 \%)$ new users for the main analysis. Of the new users of statins, 159790 (70.73\%) were prescribed simvastatin, 50328 (22.28\%) atorvastatin; 8103 (3.59\%) pravastatin; $4497(1.99 \%)$ rosuvastatin and 3204 (1.42\%) fluvastatin.

For the validation cohorts, we identified 188 practices from the OResearch database, from which we identified 118372 new users of statins and 877812 non-users of statins. We also identified a separate set of 382 practices from the THIN database. Of the 2319181 patients aged 30-84 years in the THIN database, we excluded $7743(0.33 \%)$ past users of statins and 105542 $(4.55 \%)$ current users, leaving 1923840 who had not been prescribed statins, and 282056 (12.16\%) new users for the main analysis

The baseline characteristics of the three cohorts were very similar. Table 1 compares characteristics of the OResearch derivation cohort and the THIN validation cohort (characteristics of the OResearch validation cohort are available from the authors).

As in previous studies, ${ }^{3} 1213$ the patterns of missing data supported the use of multiple imputation to replace missing values for smoking and body mass index (further details available from the authors). As previously described, ${ }^{5 a}$ compared with non-users, new users of statins in each cohort tended to be older, more likely to be male and have comorbidities such as atrial fibrillation, cardiovascular disease, peripheral vascular 
Table 1 Baseline characteristics of new users of statins and non-users of statins aged 30-84 years in the QResearch derivation and THIN validation cohorts. Figures in the tables are counts (\%) unless otherwise specified

\begin{tabular}{|c|c|c|c|c|}
\hline \multirow[b]{2}{*}{ Characteristics } & \multicolumn{2}{|c|}{ QResearch derivation cohort } & \multicolumn{2}{|l|}{ THIN validation cohort } \\
\hline & New users of statins & Non-users of statins & New users of statins & Non-users of statins \\
\hline Total & $225922(100.00)$ & $1778770(100.00)$ & $282056(100.00)$ & $1923840(100.00)$ \\
\hline Female & $104774(46.38)$ & $909423(51.13)$ & $129085(45.77)$ & $988038(51.36)$ \\
\hline Male & $121148(53.62)$ & $869347(48.87)$ & $152971(54.23)$ & $935802(48.64)$ \\
\hline Age, mean (SD) & $57.2(11.7)$ & $44.4(13.7)$ & $59.2(11.8)$ & $46(13.9)$ \\
\hline \multicolumn{5}{|l|}{ Ethnicity } \\
\hline Ethnicity recorded & 121355 (53.72) & 569466 (32.01) & 54627 (19.37) & 303572 (15.78) \\
\hline White/not recorded & $215077(95.20)$ & 1699991 (95.57) & $275014(97.50)$ & $1873857(97.40)$ \\
\hline Indian & $2861(1.27)$ & $13398(0.75)$ & $2153(0.76)$ & $10793(0.56)$ \\
\hline Pakistani & $1658(0.73)$ & $7562(0.43)$ & $776(0.28)$ & $3430(0.18)$ \\
\hline Bangladeshi & $679(0.30)$ & $3226(0.18)$ & $262(0.09)$ & $1224(0.06)$ \\
\hline Other Asian & $759(0.34)$ & $7321(0.41)$ & $809(0.29)$ & $5930(0.31)$ \\
\hline Caribbean & $1788(0.79)$ & $9853(0.55)$ & $982(0.35)$ & $4849(0.25)$ \\
\hline Black African & $834(0.37)$ & $15358(0.86)$ & $609(0.22)$ & $8967(0.47)$ \\
\hline Chinese & $316(0.14)$ & $4015(0.23)$ & $151(0.05)$ & $2206(0.11)$ \\
\hline Other ethnic group & $1950(0.86)$ & $18046(1.01)$ & $1300(0.46)$ & $12584(0.65)$ \\
\hline \multicolumn{5}{|l|}{ BMI and smoking } \\
\hline BMI recorded & 207644 (91.91) & $1341863(75.44)$ & $255544(90.60)$ & 1444453 (75.08) \\
\hline Smoking status recorded & 224982 (99.58) & $1615527(90.82)$ & $279998(99.27)$ & 1758891 (91.43) \\
\hline $\mathrm{BMI}$ and smoking status recorded & $207494(91.84)$ & $1330320(74.79)$ & $255310(90.52)$ & 1433122 (74.49) \\
\hline Body mass index, mean (SD) & $28.3(4.9)$ & $26(4.6)$ & $28.3(4.9)$ & $26.1(4.6)$ \\
\hline Non-smoker & $109406(48.43)$ & $912149(51.28)$ & $115122(40.82)$ & $795944(41.37)$ \\
\hline Ex-smoker & $74277(32.88)$ & $285271(16.04)$ & $99446(35.26)$ & $253294(13.17)$ \\
\hline Current smoker (amount not recorded) & $2286(1.01)$ & $55859(3.14)$ & $10376(3.68)$ & $239225(12.43)$ \\
\hline Current smoker (light) & $14447(6.39)$ & $116035(6.52)$ & $15406(5.46)$ & $120385(6.26)$ \\
\hline Current smoker (moderate) & $13880(6.14)$ & $142469(8.01)$ & $20494(7.27)$ & $186825(9.71)$ \\
\hline Current smoker (heavy) & $10686(4.73)$ & $103744(5.83)$ & $19154(6.79)$ & $163218(8.48)$ \\
\hline Townsend score, mean (SD) & $-0.5(3.3)$ & $-0.3(3.4)$ & $-0.5(3.1)$ & $-0.7(3.1)$ \\
\hline \multicolumn{5}{|l|}{ Comorbidity } \\
\hline Atrial fibrillation & $11656(5.16)$ & $13730(0.77)$ & $13951(4.95)$ & $15149(0.79)$ \\
\hline Congestive cardiac failure & $7457(3.30)$ & $9026(0.51)$ & $8802(3.12)$ & $9707(0.50)$ \\
\hline Cardiovascular disease & $56943(25.20)$ & $31038(1.74)$ & $77244(27.39)$ & $33838(1.76)$ \\
\hline Treated hypertension & $97782(43.28)$ & $106582(5.99)$ & $107572(38.14)$ & $99559(5.18)$ \\
\hline Chronic kidney disease $3 b+$ & $18768(8.31)$ & $17114(0.96)$ & $19156(6.79)$ & $13032(0.68)$ \\
\hline Type 1 diabetes & $2843(1.26)$ & $2633(0.15)$ & $3829(1.36)$ & $2945(0.15)$ \\
\hline Type 2 diabetes & $47703(21.11)$ & $18243(1.03)$ & $58860(20.87)$ & $18348(0.95)$ \\
\hline Rheumatoid arthritis & $3652(1.62)$ & $11762(0.66)$ & $5155(1.83)$ & $14660(0.76)$ \\
\hline Corticosteroids & $13868(6.14)$ & $44517(2.50)$ & $16816(5.96)$ & $50505(2.63)$ \\
\hline Hypothyroidism & $12378(5.48)$ & $30357(1.71)$ & $17675(6.27)$ & $38441(2.00)$ \\
\hline \multicolumn{5}{|l|}{ Clinical values } \\
\hline Systolic blood pressure, mean (SD) & $141.1(19.1)$ & $129.9(19.1)$ & $140.7(19.1)$ & $130.4(19.2)$ \\
\hline LFT recorded at baseline/before statins & $131354(58.14)$ & $162207(9.12)$ & $135793(48.14)$ & $90162(4.69)$ \\
\hline LFT recorded at follow-up & $193586(85.69)$ & $594750(33.44)$ & $224024(79.43)$ & $578686(30.08)$ \\
\hline $\begin{array}{l}\text { Creatine kinase recorded at baseline/ } \\
\text { before statins }\end{array}$ & $15724(6.96)$ & $8642(0.49)$ & $13651(4.84)$ & $4807(0.25)$ \\
\hline Creatine kinase recorded at follow-up & $62706(27.76)$ & $43333(2.44)$ & $57400(20.35)$ & $42062(2.19)$ \\
\hline
\end{tabular}

BMI, body mass index; LFT, liver function test.

disease, treated hypertension, diabetes, and chronic kidney disease. They were also more likely to have computer recorded results for liver function tests at baseline and during follow-up. For example, in the derivation cohort, $58.14 \%$ of new users of statins had a recorded LFT at baseline compared with $9.12 \%$ of non-users. At follow-up the respective figures were $85.69 \%$ and $33.44 \%$.

Overall, in the OResearch derivation cohort we identified 1969 incident cases of acute renal failure; 36541 incident cases of cataract; 15020 cases of moderate/serious liver dysfunction; 1406 incident cases of moderate/serious myopathy, of which 312 $(22.19 \%)$ were serious. The corresponding figures for the OResearch validation cohort were 1329 for acute renal failure, 23441 for cataract and 8546 for abnormal liver dysfunction and 750 for myopathy. The corresponding numbers for the THIN cohort were 2166 for acute renal failure, 27158 for cataract, 17430 for abnormal liver dysfunction and 1238 for moderate/ serious myopathy. 


\section{Myopathy}

Table 2 shows the predictor variables selected for the final model for myopathy. We found a threefold increased risk of moderate/ serious myopathy in new users of statins for women and almost sixfold increase for men (table 2).

The risk of myopathy varied by ethnic group, with Caribbean and Black African groups having the highest risks. For example, compared with men in the white reference category, Caribbean men had a sixfold increase in risk (adjusted HR 6.57, 95\% CI 4.55 to 9.48) and Black African men had nearly eightfold increased risk (adjusted HR 7.87 95\% CI 5.30 to 11.68). Risks were also increased among men in the 'Other ethnic group'.

Men prescribed corticosteroids had a twofold increase in risk and women a threefold increase. Women with type 1 diabetes had a fivefold increased risk. Hypothyroidism, type 1 diabetes, chronic liver disease and treated hypertension were significant risk factors for myopathy in women but not men.

The validation statistics (table 3) for myopathy showed that the risk prediction equation when applied to the THIN database explained $42.15 \%$ of the variation in women and $35.98 \%$ for men. The ROC statistics were 0.739 for women and 0.717 for men. The D statistic was 1.75 for women and 1.53 for men. The performance was very similar when tested on the OResearch validation sample.

\section{Acute renal failure}

Statin use was associated with a $56 \%$ increased risk of acute renal failure for women and a $61 \%$ increased risk for men (table 4). Both types of diabetes, congestive cardiac failure, corticosteroids, treated hypertension and pre-existing chronic kidney disease were all significant predictors of acute renal failure with highest risks apparent for those with type 1 diabetes and chronic kidney disease. Heavy smokers had a twofold increase in risk of acute renal failure in both men and women. We found no significant differences in risk by ethnic group.

Table 2 Adjusted HRs (95\% Cl) for moderate to severe myopathy. Models also adjusted for age and body mass index

\begin{tabular}{|c|c|c|}
\hline & $\begin{array}{l}\text { Women adjusted } \\
\text { HR ( } 95 \% \text { CI) }\end{array}$ & $\begin{array}{l}\text { Men adjusted } \\
\text { HR }(95 \% \mathrm{CI})\end{array}$ \\
\hline Non-users of statins & 1.00 & 1.00 \\
\hline New users of statins & 2.97 (2.36 to 3.74$)$ & 6.15 (5.19 to 7.30$)$ \\
\hline \multicolumn{3}{|l|}{ Ethnicity } \\
\hline White or not recorded & 1.00 & 1.00 \\
\hline Indian & $1.42(0.63$ to 3.19$)$ & $1.96(1.15$ to 3.34$)$ \\
\hline Pakistani & $1.41(0.45$ to 4.41$)$ & $1.68(0.80$ to 3.55$)$ \\
\hline Bangladeshi* & NA & $0.61(0.09$ to 4.32$)$ \\
\hline Other Asian* & NA & 0.48 (0.07 to 3.44$)$ \\
\hline Caribbean & $3.94(2.34$ to 6.64$)$ & 6.57 (4.55 to 9.48 ) \\
\hline Black African & $4.47(2.21$ to 9.04$)$ & 7.87 (5.30 to 11.68 \\
\hline Chinese & $1.44(0.20$ to 10.28$)$ & $2.10(0.52$ to 8.40$)$ \\
\hline Other ethnic group & 2.54 (1.31 to 4.92$)$ & 2.84 (1.78 to 4.53$)$ \\
\hline \multicolumn{3}{|l|}{ Comorbidity } \\
\hline Type 1 diabetes & $4.78(2.10$ to 10.86$)$ & NS \\
\hline Type 2 diabetes & 1.84 (1.39 to 2.43$)$ & 1.38 (1.12 to 1.69$)$ \\
\hline Treated hypertension & $1.62(1.31$ to 2.01$)$ & NS \\
\hline Chronic liver disease & 3.47 (1.55 to 7.78$)$ & NS \\
\hline Hypothyroidism & 1.88 (1.42 to 2.50$)$ & NS \\
\hline Corticosteroids & 3.03 (2.38 to 3.85$)$ & $2.09(1.60$ to 2.72$)$ \\
\hline
\end{tabular}

Fractional polynomial terms

women: age $^{3}$, age $^{3} \ln ($ age $)$, men: age $^{3}$, age $^{3} \ln ($ age $)$, bmi not significant in women, linear in men.

*Insufficient data in women.
The results of the validation statistics for the algorithms to predict acute renal failure (table 3) showed that the risk prediction equation when tested using the THIN database explained $59.62 \%$ of the variation in women and $59.68 \%$ for men. The ROC statistics for women were 0.831 and 0.847 for men. The D statistic was high at 2.49 in men and women, suggesting very good discrimination. The performance of the algorithms was very similar when tested on the OResearch validation sample.

\section{Moderate/serious liver dysfunction}

The significant risk factors for moderate/serious liver disease are shown in table 5. Statin use was associated with a 53\% increased risk in men and women. There was a suggestion of a dose-response effect with smoking, with heavy smokers having the highest risks of liver dysfunction compared with non-smokers. Of the comorbidities, a diagnosis of rheumatoid arthritis was the strongest predictor with an adjusted HR of 2.42 (95\% CI 2.13 to 2.76 ) for women and 1.90 (95\% CI 1.53 to 2.36 ) for men.

The validation statistics for the algorithms to predict moderate/serious liver dysfunction showed that the risk prediction equation when tested using the THIN database only explained $15.55 \%$ of the variation in women and $10.83 \%$ for men. The ROC statistics values were 0.646 for women and 0.612 for men. The D statistic was low at 0.89 for women and 0.71 for men. The performance of the algorithms was slightly improved when tested on the OResearch validation sample.

\section{Cataract}

Table 6 shows the adjusted HRs for each of the selected risk factors for cataract. Statin use was associated with a $30-32 \%$ increased risk in men and women. There were marked ethnicity differences in risk of cataract with all non-white ethnic groups having significantly higher risks than the white reference group despite adjustment for confounding variables. Smoking had a small effect on risk, whereas type 1 diabetes was associated with a more than ninefold increase in risk. Other significant predictors included type 2 diabetes, cardiovascular disease, rheumatoid arthritis (women only) and use of corticosteroids.

The results of the validation statistics for the algorithm to predict cataract (table 3) showed that the risk prediction equation when tested using the THIN database explained $59.14 \%$ of the variation in women and $59.42 \%$ for men. The ROC statistics for women were 0.878 and 0.869 for men. The D statistics were high at 2.46 for women and 2.48 for men. The performance of the algorithms was very similar when tested on the QResearch validation sample.

\section{Cardiovascular disease}

In the analysis combining the major coronary events, and, major cerebrovascular events in the seven trials reporting both outcomes presented in a recent meta-analysis, ${ }^{5}$ we calculated that, overall, these studies gave a RR of cardiovascular disease of 0.76 (95\% CI 0.67 to 0.86 ) comparing patients in the statin intervention groups with the control groups.

\section{Clinical examples}

Here are two clinical case histories illustrating the way in which 5 -year risks of each outcome would change with statin usage taking account of the patient's medical profile (also see http:// www.qintervention.org). 
Table 3 Validation statistics for each model in the THIN and QResearch validation cohorts

\begin{tabular}{|c|c|c|c|c|}
\hline & Women QResearch, mean (95\% CI) & Women THIN, mean (95\% CI) & Men OResearch, mean (95\% Cl) & Men THIN mean, $(95 \% \mathrm{CI})$ \\
\hline \multicolumn{5}{|c|}{ Acute renal failure } \\
\hline $\mathrm{R}^{2}$ statistic & $62.86(60.06$ to 65.66$)$ & $59.62(57.15$ to 62.10$)$ & $58.5(55.64$ to 61.37$)$ & 59.68 (57.44 to 61.91$)$ \\
\hline D statistic & $2.66(2.50$ to 2.82$)$ & $2.49(2.36$ to 2.62$)$ & $2.43(2.29$ to 2.57$)$ & $2.49(2.37$ to 2.61$)$ \\
\hline ROC statistic & $0.852(0.833$ to 0.872$)$ & 0.831 (0.816 to 0.846$)$ & $0.834(0.816$ to 0.853$)$ & $0.847(0.835$ to 0.859$)$ \\
\hline \multicolumn{5}{|l|}{ Cataract } \\
\hline $\mathrm{R}^{2}$ statistic & $58.70(58.06$ to 59.34$)$ & 59.14 (58.49 to 59.78$)$ & 60.55 (58.76 to 62.34$)$ & 59.42 (57.71 to 61.12$)$ \\
\hline D statistic & 2.44 (2.41 to 2.47$)$ & 2.46 (2.43 to 2.49$)$ & 2.54 (2.44 to 2.63$)$ & 2.48 (2.39 to 2.56$)$ \\
\hline ROC statistic & $0.880(0.877$ to 0.882$)$ & $0.878(0.876$ to 0.881$)$ & $0.881(0.877$ to 0.885$)$ & $0.869(0.870$ to 0.877$)$ \\
\hline \multicolumn{5}{|l|}{ Myopathy } \\
\hline $\mathrm{R}^{2}$ statistic & $40.98(40.95$ to 41.01$)$ & $42.15(42.10$ to 42.14$)$ & $38.05(38.02$ to 38.08$)$ & $35.98(35.96$ to 36.00$)$ \\
\hline D statistic & $1.71(1.61$ to 1.81$)$ & 1.75 (1.68 to 1.82$)$ & $1.6(1.51$ to 1.69$)$ & $1.53(1.47$ to 1.59$)$ \\
\hline ROC statistic & $0.727(0.691$ to 0.764$)$ & $0.739(0.716$ to 0.763$)$ & $0.743(0.714$ to 0.773$)$ & $0.717(0.697$ to 0.737$)$ \\
\hline \multicolumn{5}{|c|}{ Liver dysfunction } \\
\hline $\mathrm{R}^{2}$ statistic & $17.2(15.38$ to 19.02$)$ & 15.55 (14.31 to 16.78$)$ & $13.73(11.75$ to 15.72$)$ & $10.83(9.57$ to 12.10$)$ \\
\hline D statistic & $0.93(0.87$ to 0.99$)$ & $0.89(0.84$ to 0.92$)$ & $0.82(0.75$ to 0.89$)$ & $0.71(0.67$ to 0.76$)$ \\
\hline ROC statistic & $0.660(0.651$ to 0.670$)$ & $0.646(0.639$ to 0.652$)$ & $0.635(0.626$ to 0.644$)$ & $0.612(0.605$ to 0.619$)$ \\
\hline
\end{tabular}

Notes on understanding validation statistics:

$\mathrm{R}^{2}$ statistic shows explained variation-higher values indicate that more variation is explained.

ROC statistic is a measure of discrimination-higher values indicate better discrimination.

$\mathrm{D}$ statistic is a measure of discrimination - higher values indicate better discrimination and an increase of $\geq 0.1$ over other risk prediction models is a good marker of improved prognostic separation. ROC, receiver operating characteristic.

\section{Example 1}

A 64-year-old Caribbean man with type 2 diabetes, hypothyroidism and chronic kidney disease, who is a heavy smoker, with a body mass index of $36.7 \mathrm{~kg} / \mathrm{m}^{2}$, systolic blood pressure of $200 \mathrm{~mm} \mathrm{Hg}$, cholesterol/high-density lipoprotein ratio 4.3 would have the following 5 year risks on and off statins:

- Cardiovascular risk $30 \%$ risk off statins, $23 \%$ risk on statins;

- Acute renal failure $1.8 \%$ risk off statins, $2.9 \%$ risk on statins;

- Cataract $12.7 \%$ risk off statins, $16.4 \%$ risk on statins;

- Liver dysfunction $1.8 \%$ risk off statins, $2.8 \%$ risk on statins;

- Moderate/serious myopathy $0.7 \%$ risk off statins, $4.4 \%$ risk on statins.

Table 4 Adjusted HRs $(95 \% \mathrm{Cl})$ for acute renal failure. Models also adjusted for age and body mass index

\begin{tabular}{lll}
\hline & $\begin{array}{l}\text { Women adjusted HR } \\
\text { (95\% Cl) }\end{array}$ & $\begin{array}{l}\text { Men adjusted } \\
\text { HR (95\% Cl) }\end{array}$ \\
\hline $\begin{array}{l}\text { Non-users of statins } \\
\text { New users of statins }\end{array}$ & 1.00 & 1.00 \\
& $1.56(1.31$ to 1.86$)$ & 1.61 (1.38 to 1.86$)$ \\
$\begin{array}{l}\text { Townsend score } \\
\text { (5 unit increase) }\end{array}$ & $1.25(1.15$ to 1.35$)$ & 1.20 (1.10 to 1.30$)$ \\
Smoking status & & \\
Non-smoker & 1.00 & 1.00 \\
Ex-smoker & $1.23(1.05$ to 1.45$)$ & $1.14(0.99$ to 1.31$)$ \\
Light smoker & $1.25(0.91$ to 1.71$)$ & $1.60(1.29$ to 1.97$)$ \\
Moderate smoker & $1.36(1.03$ to 1.80$)$ & $1.52(1.18$ to 1.96$)$ \\
Heavy smoker & $1.83(1.33$ to 2.51$)$ & $1.85(1.43$ to 2.38$)$ \\
& & \\
Comorbidity & & \\
Type 1 diabetes & $4.22(1.57$ to 11.38$)$ & $5.89(3.23$ to 10.77$)$ \\
Type 2 diabetes & $1.99(1.62$ to 2.44$)$ & $1.71(1.43$ to 2.03$)$ \\
Congestive cardiac failure & $2.30(1.79$ to 2.96$)$ & $2.59(2.10$ to 3.19$)$ \\
Corticosteroids & $1.60(1.30$ to 1.97$)$ & $1.77(1.44$ to 2.16$)$ \\
Treated hypertension & $1.38(1.18$ to 1.62$)$ & $1.40(1.22$ to 1.61$)$ \\
Chronic kidney disease $3 b+$ & $2.81(2.33$ to 3.39$)$ & $2.76(2.28$ to 3.33$)$ \\
\hline
\end{tabular}

Fractional polynomial terms women: $\mathrm{age}^{-1}, \mathrm{age}^{-0.5}, \mathrm{bmi}^{-2}, \mathrm{bmi}^{-2} \ln (\mathrm{bmi})$, men: age ${ }^{-1}$, age $^{-1} \ln ($ age $)$, bmi $^{-2}$, bmi $^{-1}$.

\section{Example 2}

A 60-year-old white women, with rheumatoid arthritis, currently prescribed corticosteroids, heavy smoker, with a systolic blood pressure of $190 \mathrm{~mm} \mathrm{Hg}$, cholesterol/high-density lipoprotein ratio of 7 ; body mass index $33.1 \mathrm{~kg} / \mathrm{m}^{2}$ would have the following 5 -year risks on and off statins:

- Cardiovascular risk $21 \%$ risk off statins, $16 \%$ risk on statins;

- Acute renal failure $0.4 \%$ risk off statins, $0.7 \%$ risk on statins;

- Cataract $7.1 \%$ risk off statins, $9.1 \%$ risk on statins;

- Liver dysfunction $5.4 \%$ risk off statins, $8.2 \%$ risk on statins;

- Moderate/serious myopathy $0.2 \%$ risk off statins, $0.7 \%$ risk on statins.

For these examples, the mean deprivation score has been used. A postcode in a deprived area would increase the risks of cardiovascular disease (men and women) and acute renal failure (men and women) and cataract (men only). Also note that in the example, 5-year risks of cardiovascular disease have been used for consistency, although 10-year risks are also available.

\section{Thresholds and risk stratification}

Since these are new algorithms and there are no established thresholds, we used the 90th centile of risk from the full OResearch validation population to define a high-risk group (ie, the top $10 \%$ at highest risk) for each outcome for men and women. We then determined the numbers and proportion of incident cases over 5 years in the OResearch validation cohort which fell within the top tenth of risk.

For example, for acute renal failure in women aged 30-84, the 90 th centile defined a high-risk group with a 5-year risk score of $>0.24 \%$. There were 268 new cases of acute renal failure within this, which accounted for $58.77 \%$ of all new cases of acute renal failure in the population. The 90th centile for acute renal failure for men was $0.35 \%$, which accounted for $50.08 \%$ of new cases over 5 years. For myopathy in women, then patients with a risk score of $>0.14 \%$ would be included within the $10 \%$ at highest risk. Of all the new cases of myopathy which occurred in women in the OResearch validation cohort over 5 years, $32.24 \%$ were in this group. The corresponding figures for myopathy in 
Table 5 Adjusted HR (95\% Cl) for moderate/serious liver dysfunction. Models also adjusted for age and body mass index

\begin{tabular}{|c|c|c|}
\hline & $\begin{array}{l}\text { Women adjusted } \\
\text { HR }(95 \% \mathrm{Cl})\end{array}$ & $\begin{array}{l}\text { Men adjusted } \\
\text { HR (95\% CI) }\end{array}$ \\
\hline Non-users of statins & 1.00 & 1.00 \\
\hline New users of statins & $1.53(1.41$ to 1.66$)$ & 1.53 (1.42 to 1.66$)$ \\
\hline Townsend score (5 unit increase) & 0.93 (0.98 to 0.99 ) & $1.02(1.00$ to 1.01$)$ \\
\hline \multicolumn{3}{|l|}{ Ethnicity } \\
\hline White or not recorded & 1.00 & 1.00 \\
\hline Indian & $0.94(0.7$ to 1.26$)$ & $1.16(0.90$ to 1.50$)$ \\
\hline Pakistani & 1.45 (1.05 to 2.00$)$ & $1.18(0.85$ to 1.63$)$ \\
\hline Bangladeshi & $1.41(0.8$ to 2.50$)$ & 1.31 (0.81 to 2.11$)$ \\
\hline Other Asian & $1.62(1.1$ to 2.39$)$ & $1.43(0.96$ to 2.13$)$ \\
\hline Caribbean & 0.51 (0.34 to 0.76$)$ & $0.86(0.62$ to 1.20$)$ \\
\hline Black African & 0.74 (0.49 to 1.12$)$ & 0.56 (0.36 to 0.87$)$ \\
\hline Chinese & 1.40 (0.81 to 2.42$)$ & 1.29 (0.73 to 2.28$)$ \\
\hline Other ethnic group & 0.94 (0.72 to 1.24$)$ & 1.17 (0.93 to 1.49$)$ \\
\hline \multicolumn{3}{|l|}{ Smoking status } \\
\hline Non-smoker & 1.00 & 1.00 \\
\hline Ex-smoker & $1.06(1.00$ to 1.13$)$ & 1.14 (1.08 to 1.21$)$ \\
\hline Light smoker & $1.12(1.01$ to 1.24$)$ & 1.21 (1.12 to 1.32$)$ \\
\hline Moderate smoker & $1.23(1.13$ to 1.34$)$ & 1.19 (1.09 to 1.29$)$ \\
\hline Heavy smoker & $1.36(1.23$ to 1.51$)$ & $1.34(1.23$ to 1.45$)$ \\
\hline \multicolumn{3}{|l|}{ Comorbidity } \\
\hline Type 1 diabetes & 2.15 (1.51 to 3.07$)$ & 1.52 (1.09 to 2.13$)$ \\
\hline Type 2 diabetes & $1.13(1.00$ to 1.27$)$ & 1.45 (1.31 to 1.60$)$ \\
\hline Cardiovascular disease & $1.14(1.02$ to 1.27$)$ & 1.22 (1.11 to 1.34$)$ \\
\hline Treated hypertension & 1.23 (1.15 to 1.32$)$ & 1.40 (1.31 to 1.51$)$ \\
\hline Rheumatoid arthritis & 2.42 (2.13 to 2.76$)$ & 1.90 (1.53 to 2.36$)$ \\
\hline Corticosteroids & 1.47 (1.34 to 1.61$)$ & 1.44 (1.29 to 1.62$)$ \\
\hline
\end{tabular}

Fractional polynomial terms

women; age ${ }^{2}$, age $^{2} \ln ($ age $), \mathrm{bmi}^{3}, \mathrm{bmi}^{3} \ln (\mathrm{bmi})$, men $\mathrm{age}^{-2}, \mathrm{age}^{3}, \mathrm{bmi}^{-2}, \mathrm{bmi}^{-2} \ln (\mathrm{bmi})$.

men were $0.35 \%$ for the 90 th centile, which accounted for $32.94 \%$ of cases in men. For liver dysfunction the 90th centile cut-off point was $1.34 \%$ in women and $1.44 \%$ in men and this accounted for $19.43 \%$ and $17.07 \%$ of cases, respectively. For cataract, the 90 th centile cut-off point was $9.38 \%$ in women and $5.93 \%$ in men and this accounted for $50.24 \%$ of new cases in women and $57.79 \%$ of new cases in men.

\section{Calibration}

Figure 1 shows the mean predicted and observed risk for each outcome across each tenth of predicted risk as a measure of calibration. The algorithms to predict cataract and acute renal failure were well calibrated in men and women as was the algorithm for myopathy in women. There was a small degree of overprediction for the algorithm to predict myopathy in men. The algorithm to predict liver dysfunction was the least well calibrated.

\section{Numbers needed to treat and numbers needed to harm}

Table 7 shows the numbers of patients needed to treat and numbers needed to harm for each outcome among patients aged 35-74 years at high risk of cardiovascular disease as defined based on the ORISK2 10-year cardiovascular risk score. The NNT with any statin to prevent one case of cardiovascular disease for women over 5 years was 37 (95\% CI 27 to 64) and 33 ( $95 \%$ CI 24 to 57 ) for men. The NNH for an additional case of acute renal failure in women over 5 years was 434 (95\% CI 284
Table 6 Adjusted HR $(95 \% \mathrm{Cl})$ for cataract. Models also adjusted for age and body mass index

\begin{tabular}{lll}
\hline & $\begin{array}{l}\text { Women adjusted } \\
\text { HR (95\% Cl) }\end{array}$ & $\begin{array}{l}\text { Men adjusted } \\
\text { HR (95\% Cl) }\end{array}$ \\
\hline $\begin{array}{l}\text { Non-users of statins } \\
\text { New users of statins }\end{array}$ & 1.00 & 1.00 \\
& $1.30(1.26$ to 1.35$)$ & 1.32 (1.26 to 1.37$)$ \\
Townsend score & NS & $1.05(1.02$ to 1.08$)$ \\
(5 unit increase) & & \\
& & \\
Ethnicity & 1.00 & 1.00 \\
White or not recorded & $2.48(2.18$ to 2.81$)$ & $2.59(2.24$ to 3.00$)$ \\
Indian & $2.27(1.86$ to 2.77$)$ & $2.40(1.94$ to 2.97$)$ \\
Pakistani & $4.32(3.34$ to 5.58$)$ & $2.77(2.05$ to 3.74$)$ \\
Bangladeshi & $2.13(1.62$ to 2.80$)$ & $2.72(2.05$ to 3.62$)$ \\
Other Asian & $1.80(1.54$ to 2.10$)$ & $2.10(1.77$ to 2.49$)$ \\
Caribbean & $3.13(2.56$ to 3.83$)$ & 3.52 (2.81 to 4.41$)$ \\
Black African & $2.70(2.00$ to 3.65$)$ & $2.07(1.37$ to 3.12$)$ \\
Chinese & $1.40(1.19$ to 1.64$)$ & 1.29 (1.05 to 1.58$)$ \\
Other ethnic group &
\end{tabular}

Smoking status

Non-smoker

Ex-smoker

Light smoker

Moderate smoker

1.09 (1.05 to 1.12$)$

1.01 (0.94 to 1.08 )

$1.02(0.95$ to 1.09$)$

1.11 (1.02 to 1.21$)$

1.00

1.09 (1.05 to 1.13 )

1.01 (0.95 to 1.08$)$

1.05 (0.97 to 1.14$)$

Heavy smoker

$1.15(1.06$ to 1.26$)$

Comorbidity

Cardiovascular disease $\quad 1.11(1.06$ to 1.16$) \quad 1.13(1.08$ to 1.18$)$

Type 1 diabetes

Type 2 diabetes

Rheumatoid arthritis

Corticosteroids

10.02 (8.23 to 12.19 )

9.77 (8.13 to 11.74

2.44 (2.32 to 2.56 )

ns

1.16 (1.07 to 1.27$)$

1.47 (1.41 to 1.54

1.58 (1.49 to 1.68$)$

Atrial fibrillation

$1.16(1.09$ to 1.25$)$

1.23 (1.15 to 1.32 )

Fractional polynomial terms

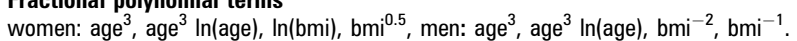

to 783). In women for moderate/severe myopathy the $\mathrm{NNH}$ was 259 (95\% CI 186 to 375), for moderate to severe liver dysfunction the NNH was 136 (95\% CI 109 to 175) and for cataract it was 33 (95\% CI 28 to 38). Overall, the NNH/NNTs for men were similar to those for women except for myopathy where the $\mathrm{NNH}$ was 91 (95\% CI 74 to 112). This is lower than for women mainly owing to the higher HRs in men.

Table 7 also shows the estimated numbers of extra cases or cases prevented per 10000 patients treated with statins at both thresholds. For example, there would be 271 fewer cases $(95 \% \mathrm{CI}$ 157 to 374) of cardiovascular disease for every 10000 women using the $20 \%$ threshold compared with 228 fewer cases (95\% CI 132 to 315 ) using the $15 \%$ threshold.

\section{DISCUSSION}

\section{Summary of key findings}

We have developed and externally validated four new risk prediction algorithms designed to predict the absolute risk of clinical outcomes which might change with statin usage in a large representative primary care population. The algorithms to predict acute renal failure, moderate/serious myopathy and cataract performed well in both validation cohorts and could be used to identify patients at increased risk of these clinical outcomes so that patients can be monitored more closely. While the algorithms have specifically been designed to help inform 
Figure 1 Predicted to observed risk of each outcome using the QResearch database.
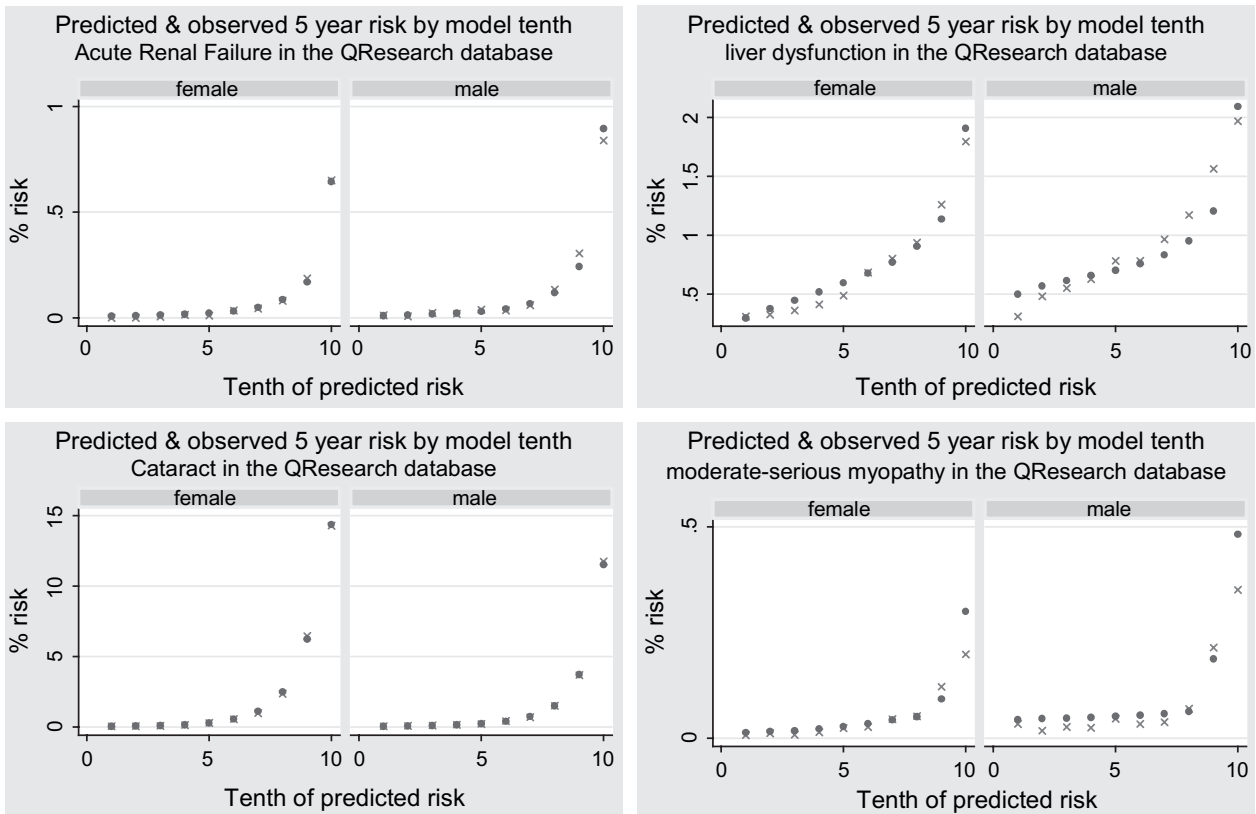

Predicted \& observed 5 year risk by model tenth moderate-serious myopathy in the QResearch database

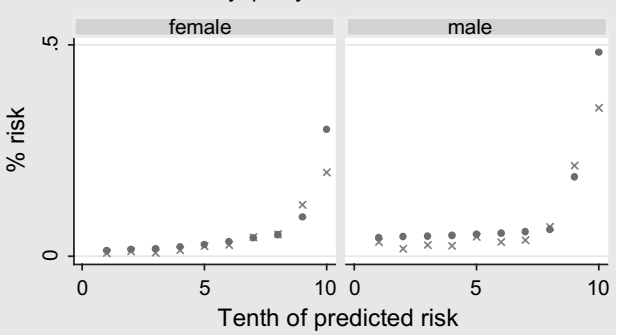

treatment choices on risks and benefits of statins, they can also be applied to the general population. The algorithm to predict risk of liver dysfunction did not perform sufficiently well to justify its use in patient care. However, given the increased risk of liver dysfunction in statin users overall, all statin users should have liver function tests performed according to existing guidelines.

\section{Comparison with previous studies}

Our study has good face validity since it has been conducted in the setting where the majority of patients in the UK are assessed, treated and followed up. While other studies have examined adverse effects of statins using meta-analyses of clinical trials ${ }^{6}{ }^{26}$ or observational data, ${ }^{7}$ we think ours is the first to develop algorithms to individualise risk for the patient. ${ }^{7}$ Our study adds to previous studies by analysis of a much larger population over a longer time period.

We included acute renal failure as an outcome because of concerns published in the Lancet $^{27}$ and on the FDA website ${ }^{28}$ together with reports of proteinuria in patients prescribed rosuvastatin. ${ }^{29}$ Although our definition of adverse outcomes is broadly consistent with that used elsewhere ${ }^{26}$ studies vary in their definition of severity. Our definition of liver dysfunction is similar to that used in other guidelines ${ }^{11}$ and studies ${ }^{26}$ and is the level at which treatment should be discontinued. ${ }^{11}$ Our definition of acute renal failure was based on presence of a computerrecorded diagnostic code, whereas other studies ${ }^{7}$ have also required a computer code to indicate hospital admission. We think our definition is valid since it would be highly unusual for treatment of a patient with acute renal failure to be managed at home. Our definition of moderate/serious myopathy was based on computer-recorded diagnosis of myopathy or a creatine kinase of four or more times the upper limit of normal, which is similar to the value used in some studies, ${ }^{30} 31$ though other studies have used 10 or more times the upper limit of normal. ${ }^{7}$
We think our outcome definition is clinically useful since it will better identify a population at risk at a time when closer assessment and interventions might help reduce progression to more severe disease.

Also, we have been able to confirm associations between statin use and adverse outcomes including myopathy, ${ }^{26} 30-32$ liver dysfunction ${ }^{26}$ and acute renal failure ${ }^{26}$ which are consistent with those elsewhere and which further increases face validity. Other studies found inconsistent results for the association between statin use and cataract. ${ }^{10}{ }^{33}$ For example, one study of statin use reported a decrease in risk of nuclear cataracts with a tendency for an increased risk of cortical cataracts, ${ }^{10}$ while another early study of pravastatin found no association but only studied 14 patients. ${ }^{33}$ Smeeth et al reported an increased risk of cataract of similar magnitude to that obtained in our study which did not vary by type, dose or duration of use. ${ }^{34}$ This increase was largely explained by an increase in consultation rates, which suggests that the increased risk may be more an ascertainment bias than a true biological effect.

We have also been able to provide some new information on the association between ethnicity and risk of each outcome. We found that Black Caribbean and Black African patients have the greatest risk of myopathy-six- to eightfold higher risks for men and four- to fivefold higher risks for in women compared with the White reference population. This is interesting given recent studies suggesting an association for genetic variants in statininduced myopathy. ${ }^{31}$

\section{Strengths and weaknesses}

The strengths and weaknesses of using GP databases for the development and validation of clinical risk prediction algorithms has been described in detail elsewhere. ${ }^{3}{ }^{18}$ In summary, key strengths include size, duration of follow-up, representativeness, and lack of selection, recall and respondent bias. Limitations include lack of formally adjudicated outcomes, information bias, 
Table 7 Numbers needed to harm (NNH) or numbers needed to treat (NNT) and numbers of extra or prevented cases, for each outcome over 5 years in patients aged 35-74 free of cardiovascular disease at baseline with QRISK2 score of $20 \%+$ (table reproduced from the BMJ ${ }^{5 a}$ )

\begin{tabular}{ll}
\hline NNH or NNT & $\begin{array}{l}\text { Estimated number of extra } \\
\text { cases (or cases prevented) } \\
(95 \% \mathrm{Cl})\end{array}$ \\
per 10000 patients treated
\end{tabular}

Potential benefits in women

Cardiovascular disease $\quad-37(-64$ to -27$) \quad-271(-374$ to -157$)$

Potential harms in women

Acute renal failure

Cataract

Liver dysfunction

Myopathy

434 (284 to 783 )

33 (28 to 38$)$

136 (109 to 175$)$

259 (186 to 375$)$

Potential benefits in men

Cardiovascular disease

$-33(-57$ to -24$)$

$-301(-417$ to -174$)$

Potential harms in men

Acute renal failure

Cataract

346 (245 to 539$)$

$52(44$ to 63$)$

Liver dysfunction

$142(115$ to 180$)$

91 (74 to 112)

Myopathy

Note: negative numbers indicate numbers needed to treat or cases prevented and positive numbers indicate numbers needed to harm or extra cases.

potential for missing data, unmeasured confounding. While we are reliant on accuracy of information recorded by primary care doctors, we think that the quality of information is likely to be good since previous studies have validated similar outcomes and exposures using questionnaire data, and found levels of completeness and accuracy to be high. ${ }^{7}$ Nonetheless, it is possible that there is some overlap between some of the outcomes-for example, some cases of acute renal failure might be due to rhabdomyolysis.

Ascertainment bias might account for the increased risk of cataract since patients prescribed statins may be consulting their GP more frequently thereby increasing the opportunity for patients to report visual problems and be examined. ${ }^{34}$

A key strength of our study is that we have developed the algorithms in one cohort and validated it in two independent cohorts, each of which is representative of the patients likely to be offered statins. The size of our study is particularly important since some of the adverse events we have studied are relatively uncommon and so associations with statin use would not necessarily be detected in a much smaller clinical trial or metaanalysis.

While the performance of three of the algorithms was very good, the liver dysfunction algorithm did not perform sufficiently well to justify its clinical use. This might be due to the non-specific nature of liver dysfunction, which has many potential causes (and so is particularly susceptible to unmeasured confounding). In addition, it might be due to misclassification of the outcome since a large proportion of patients did not have an alanine transaminase value recorded in their computer record. This is despite recommendations that all patients have liver function tests 3 and 12 months after starting statin treatment. The algorithm could be remodelled once the recording of liver function tests is more complete.

Finally, while our study covers a broad age range (up to 84 years), we have not studied the very elderly, who may be prescribed statins and may be more susceptible to adverse effects.

\section{What is already known on the subject}

Meta-analyses of primary prevention trials indicate that statins reduce the risk of cardiovascular disease particularly in high-risk patients.

- Meta-analyses provide valuable information on effectiveness but tend not to have representative samples or sufficient detail, duration of follow-up or power to fully assess unintended effects.

- There is a lack of information on the unintended effects of statins in representative primary care populations, although such drugs are prescribed in large volumes for long periods of time.

\section{What this study adds}

We have developed and validated four new risk prediction algorithms that can be used to identify patients at high risk of clinical outcomes which change with statin treatment.

- Three algorithms performed well in both validation datasets. These were acute renal failure, moderate/serious myopathy and cataract.

- The performance of the algorithm to predict liver dysfunction was poor and more research is needed to produce a better algorithm.

- The algorithms to predict acute renal failure, moderate/serious myopathy and cataract could be used to identify patients at high risk of adverse effects so that patients can be monitored more closely. While the algorithms have specifically been designed to help inform treatment choices on risks and benefits of statins, they can also be applied to the general population.

\section{Clinical implications}

The algorithms to predict risk of cataract, moderate/serious myopathy and acute renal failure could potentially be used within a consultation to assess and discuss the balance of risks and benefits at the start of statin treatment. They can also be used to identify patients at increased risk of adverse effects so that these patients can be monitored more closely. Patients at increased risk of acute renal failure could have lower doses of statins ${ }^{6}$ and more regular assessment of glomerular filtration rates, especially in the first year after starting treatment. ${ }^{6}$ Patients at high risk of myopathy could be warned to report any unusual muscular aches and pains and could be asked about these at their follow-up consultations. Clinicians could also be alerted of patients at high risk of myopathy via the computer system and be prompted to measure creatine kinase levels. Although ascertainment bias might account for the increased risk of cataract, nonetheless, such patients appear to be at increased risk and could be advised to report any visual symptoms and be followed-up by an optician if they are not already receiving regular follow-up because of a comorbid condition such as diabetes.

\section{Summary}

We have developed and validated four new risk prediction algorithms designed to quantify the absolute risk of moderate/ serious myopathy, acute kidney failure, cataract and moderate/ 
serious liver dysfunction associated with statin use in a large representative primary care population. Three of the algorithms could be used to identify patients at high risk of these clinical outcomes so that patients can be monitored more closely. Also, the algorithms can be used within a consultation to assess the balance of risks and benefits at the start of statin treatment.

Acknowledgements We acknowledge the contribution of EMIS practices who contribute to the QResearch and to David Stables (medical director, EMIS) for his expertise in establishing, developing and supporting the database. We also acknowledge the contribution of practices using InPractice Systems and to EPIC for the provision of THIN data.

Funding The project was undertaken by ClinRisk Ltd. There was no external funding. Other Funders: work undertaken by ClinRisk Ltd.

Competing interests $\mathrm{JHC}$ is professor of clinical epidemiology at the University of Nottingham and co-director of QResearch-a not-for-profit organisation, which is a joint partnership between the University of Nottingham and EMIS (leading commercial supplier of IT for $60 \%$ of general practices in the UK). JHC is also director of ClinRisk Ltd which produces software to ensure the reliable and updatable implementation of clinical risk algorithms within clinical computer systems to help improve patient care. CC is associate professor of medical statistics at the University of Nottingham and a consultant statistician for ClinRisk Ltd. This work and any views expressed within it are solely those of the co-authors and not of any affiliated bodies or organisations.

Ethics approval This study was conducted with the approval of the Trent Multi-Centre Ethics Committee as part of the QResearch approval process.

Contributors JHC initiated the study, undertook the literature review, data extraction, data manipulation and primary data analysis and write the first draft of the paper. CC contributed to the design, analysis, interpretation and drafting of the paper.

Provenance and peer review Not commissioned; externally peer reviewed.

\section{REFERENCES}

1. British Heart Foundation. Coronary heart disease statistics. London: British Heart Foundation, 2007.

2. Department of Health. Putting prevention first: vascular checks: risk assessment and management. In: Health Do, ed. London: Department of Health, 2008;15. http://www. dh.gov.uk/en/Publicationsandstatistics/Publications/PublicationsPolicyAndGuidance/ DH 083822 .

3. Hippisley-Cox J, Coupland C, Vinogradova Y, et al. Predicting cardiovascular risk in England and Wales: prospective derivation and validation of QRISK2. BMJ 2008;336:1475-82. bmi.39609.449676.25.

4. Jackson R, Marshall R, Kerr A, et al. ORISK or Framingham for predicting cardiovascular risk? BMJ 2009;339:b2673. doi:10.1136/bmi.b2673.

5. Brugts JJ, Yetgin T, Hoeks SE, et al. The benefits of statins in people without established cardiovascular disease but with cardiovascular risk factors: meta-analysis of randomised controlled trials. BMJ 2009;338:b2376.

5a. Hippisley-Cox $\mathbf{J}$, Coupland CA. Unintended effects of statins in men and women in England and Wales: population based cohort study using the QResearch database. BMJ (2010) in press.

6. Maron DJ, Fazio S, Linton MF. Current Perspectives on Statins. Circulation 2000;101:207-13.

7. García-Rodríguez LA, Massó-González EL, Wallander M-A, et al. The safety of rosuvastatin in comparison with other statins in over 100000 statin users in UK primary care. Pharmacoepidemiology and Drug Safety 2008:17:943-52.
8. Nichols G, Koro G. Does statin therapy initiation increase the risk for myopathy? An observational study of 32,225 diabetic and non diabeic patients. Clin Therap 2007:29:1761-70.

9. Andrade SE, Graham DJ, Staffa JA, et al. Health plan administrative databases can efficiently identify serious myopathy and rhabdomyolysis. Journal of Clinical Epidemiology 2005:58:171-4.

10. Klein BEK, Klein R, Lee KE, et al. JAMA 2006;295:2752-58.

11. NICE clinical guideline 64. Lipid modification-Cardiovascular risk assessment and the modification of blood lipids for the primary and secondary prevention of cardiovascular disease. In: NICE, ed. London: NICE, 2008.

12. Hippisley-Cox J, Coupland C, Robson J, et al. Predicting risk of type 2 diabetes in England and Wales: prospective derivation and validation of ODScore. BMJ 2009;338:b880

13. Hippisley-Cox J, Coupland C. Predicting risk of osteoporotic fracture in men and women in England and Wales: prospective derivation and validation of OFractureScores. BMJ 2009;339:b4229.

14. Graham DJ, Staffa JA, Shatin D, et al. Incidence of hospitalized rhabdomyolysis in patients treated with lipid-lowering drugs. JAMA 2004;292:2585-90.

15. Thompson PD, Clarkson PM, Rosenson RS. An Assessment of Statin Safety by Muscle Experts. The American Journal of Cardiology 2006:97(8 Suppl 1):S69-76.

16. Thompson PD, Clarkson P, Karas RH. Statin-associated myopathy. JAMA 2003:289:1681-90.

17. Hippisley-Cox J, Coupland C, Vinogradova $Y$, et al. Derivation and validation of ORISK, a new cardiovascular disease risk score for the United Kingdom: prospective open cohort study. BMJ 2007;335:136.

18. Hippisley-Cox J, Coupland C, Vinogradova $Y$, et al. Performance of the QRISK cardiovascular risk prediction algorithm in an independent UK sample of patients from general practice: a validation study. Heart 2008:94:34-9.

19. Collins GS, Altman DG. An independent external validation and evaluation of QRISK cardiovascular risk prediction: a prospective open cohort study. BMJ 2009;339:b2584

20. Schafer J, Graham J. Missing data: our view of the state of the art. Psychological Methods 2002; 7:147-77.

21. Group TAM. Academic medicine: problems and solutions. British Medical Journal 1989:298:573-9.

22. Steyerberg EW, van Veen M. Imputation is beneficial for handling missing data in predictive models. J Epidemiol Community Health 2007;60:979.

23. Moons KGM, Donders RART, Stijnen T, et al. Using the outcome for imputation of missing predictor values was preferred. J Epidemiol Community Health 2006:59:1092.

24. Royston P, Ambler G, Sauerbrei W. The use of fractional polynomials to model continuous risk variables in epidemiology. Int J Epidemiol 1999;28:964-74.

25. Royston $\mathbf{P}$, Sauerbrei W. A new measure of prognostic separation in survival data. Stat Med 2004;23:723-48.

26. Tolman KG. The liver and lovastatin. The American Journal of Cardiology 2002:89:1374-80.

27. Wolfe SM. Dangers of rosuvastatin identified before and after FDA approval. The Lancet 2004;363:2189-90.

28. FDA. Information for healthcare professionals: Crestor (Rosuvastatin calcium). Rockville: FDA, 2005.

29. Shepherd J, Hunninghake DB, Stein EA, et al. Safety of rosuvastatin. The American Journal of Cardiology 2004;94:882-8.

30. Molokhia M, McKeigue P, Curcin V, et al. Statin induced myopathy and myalgia: time trend analysis and comparison of risk associated with statin class from 1991-2006. PLOS One 2008;3:e2522

31. The SEARCH Collaborative Group. SLCO1B1 variants and statin-induced myopathy —a genomewide study. N Engl J Med 2008;359:789-99.

32. Sathasivam S, Lecky B. Statin induced myopathy. BMJ 2008;337:a2286

33. Behrens-Baumann W, Thiery J, Fieseler HG, et al. Pravastatin-ocular side effects after a two year follow-up? Lens Eye Toxic Res 1990;7:311-18.

34. Smeeth L, Hubbard R, Fletcher AE. Cataract and the use of statins: a case-control study. OJM 2003:96:337-43. 